\title{
A histological study of hyaline deposits in laryngeal, aural, and nasal polyps and their differentiation from amyloid
}

\author{
J. C. MCALPINE ${ }^{1}$ AND J. D. BANCROFT \\ From the Department of Pathology, Institute of Laryngology and Otology, London
}

SYNOPSIS Sections of hyalinized laryngeal polyps stained with haematoxylin and eosin or Congo red can easily be confused with localized amyloid of the larynx. Several histological methods were applied to 61 specimens containing hyaline deposits or hyalinized tissues which included 36 laryngeal polyps. The results were compared with seven cases of localized amyloidosis of the upper respiratory tract and four cases of secondary amyloidosis.

The hyaline deposits gave a pattern of results which were different from amyloid. They showed no metachromasia, low affinity for Congo red, weak birefringence in an unstained state, no birefringence if stained with Congo red, weak or negative fluorescence if stained with Thioflavin $T$, low resistance to pepsin digestion, positive phosphotungstic acid haematoxylin staining areas, and a high affinity for the picric acid component of van Gieson stain. Hyalinized collagen closely resembles amyloid, but it can be excluded by a van Gieson stain.

No evidence of amyloid formation was found in areas of hyaline deposits in the larynx. This supports the view that localized amyloidosis of the laryn $\mathrm{x}$ and hyalinized laryngeal polyps are different pathological entities.

In a recent study of localized amyloidosis of the respiratory tract, McAlpine, Radcliffe, and Friedmann (1963) discussed the methods of distinguishing amyloid from other varieties of hyaline substances. They concluded that a homogenous acellular eosinophilic substance which showed metachromasia, strong affinity for Congo red, birefringence and dichroism if stained with Congo red, no birefringence or dichroism if unstained, strong fluorescence in ultra-violet light if stained with Thioflavin $T$, and a high degree of resistance to pepsin extraction was characteristic of amyloid. However, it was pointed out that the Thioflavin $T$ method had not been subjected to an extensive control study using hyaline (non-amyloid) deposits.

In localized laryngeal amyloidosis the main histological differential diagnosis is from areas of hyalinization occurring in laryngeal polyps. In many cases these areas are indistinguishable from amyloid when stained with haematoxylin and eosin. Further they also show a strong affinity for Congo red, which

${ }^{1}$ Present address: Department of Pathology, Makerere University College, P.O. Box 2072, Kampala, Uganda.

Received for publication 19 August 1963. has led to errors of diagnosis and confusion in the literature. The main purpose of the present study was to apply the methods used by McAlpine et al. (1963) to specimens of hyalinized collagen and hyaline deposits occurring in laryngeal, aural, and nasal polyps, in order to assess their value for differentiating amyloid from hyaline deposits. The other purpose was to examine the laryngeal hyaline deposits for amyloid, so as to look for evidence of a possible transformation of hyaline material into true amyloid.

\section{MATERIALS AND METHODS}

Specimens were only selected if the haematoxylin-andeosin-stained sections had been reported on as showing some degree of 'hyaline degeneration', "hyalinization', or contained 'hyaline deposits' which resembled amyloid. A few specimens were also included which contained areas of hyalinized collagen (Fig. 1) or thickened basement membranes. The sources and various types of hyaline are given in Table I. The laryngeal polyps all showed areas of perivascular, intravascular, and interstitial hyaline material (Figs. 2 and 3). Several also showed a subepithelial acellular hyaline deposit which was often homogeneous but never showed the 'flake' form, round 


\section{TABLE I}

DETAILS OF SPECIMENS CONTAINTNG HYALINIZED TISSUES OR HYALINE DEPOSITS

\begin{tabular}{|c|c|c|}
\hline Site & No. & Type of Hyalinization or Hyaline Deposit \\
\hline Larynx & 36 & $\begin{array}{l}\text { Vocal cord polyps showing areas of massive } \\
\text { hyalinization }\end{array}$ \\
\hline Ear & 7 & $\begin{array}{l}\text { Six polyps with areas of interstitial hyaline } \\
\text { material and hyalinized vessels, one hyalinized } \\
\text { fibroma }\end{array}$ \\
\hline Nose & 9 & $\begin{array}{l}\text { Five polyps with areas of interstitial hyaline } \\
\text { material, of which four had hyalinized vessels }\end{array}$ \\
\hline Pharynx & 3 & Hyalinized collagen \\
\hline Antrum & 3 & $\begin{array}{l}\text { Two biopsies of mucosa with thickened hyalinized } \\
\text { basement membranes } \\
\text { One specimen of diffuse subepithelial hyaline } \\
\text { material }\end{array}$ \\
\hline $\begin{array}{l}\text { Trachea } \\
\text { Neck }\end{array}$ & $\begin{array}{l}1 \\
2\end{array}$ & $\begin{array}{l}\text { Mucosa with hyalinized basement membrane } \\
\text { One mass of hyalinized fibrous tissue. One skin } \\
\text { biopsy with hyalinized collagen }\end{array}$ \\
\hline
\end{tabular}

laminated forms, or the foreign-body giant-cell reaction previously observed with deposits of amyloid (McAlpine et al., 1963). The hyalinized blood vessels closely resembled amyloid deposition in vessel walls (Fig. 4).

All material consisted of formalin-fixed, paraffinembedded tissue removed at operation or as diagnostic biopsy specimens. The control amyloid material consisted of seven cases of localized amyloidosis of the respiratory tract (cases 1 to 6 of McAlpine et al., 1963 and the case of McAlpine and Fuller, 1964) and blocks from four cases of generalized secondary amyloidosis.

Paraffin sections $5 \mu$ thick were mounted on to glass slides with albumin adhesive and dried overnight in a hot air oven. The sections were stained with Mayer's haematoxylin and eosin; van Gieson's stain (Pearse, 1960); Mallory's phosphotungstic acid haematoxylin (P.T.A.H.) (Pearse, 1960); and for metachromasia with methyl violet 2B (Crystal violet, colour index 681, G. T. Gurr, Ltd.), using the second method given by Pearse (1960), and staining for 16 hours. The sections stained for metachromasia were examined immediately after differentiation without mounting in glycerine jelly. Two Congo red methods were selected after a preliminary study of several techniques, namely, Highman's method (1946) and Freudenthal's technique as given by Culling (1957), using 20 minutes' treatment with formalin in the second stage. The latter method was chosen so that a study of the behaviour of Congo red-stained hyaline deposits and other tissues could be made in polarized light. The pepsin extraction procedure and the Thioflavin $T$ methods are given in the previous study (McAlpine et al., 1963). Unstained, thoroughly de-waxed sections were mounted in fluorescent-free mountant and used for the ultraviolet and polarization microscopy controls. Sections stained with Thioflavin $\mathrm{T}$ were examined in ultra-violet light, and sections stained with both Congo red methods were examined in polarized light.

\section{RESULTS}

METACHROMASIA None of the specimens showed metachromasia in the interstitial, intravascular, or perivascular hyaline deposits in the laryngeal, aural, and nasal polyps, but three specimens of hyalinized collagen showed a few areas of weak metachromasia.

CONGO RED AFFINITY BY HIGHMAN'S METHOD The hyaline material in 40 polyps from the larynx, ear, 을 and nose showed no staining. In five laryngeal polyps a faint orange-red staining was obtained in the $\Phi$ hyaline deposits, and the hyaline deposits around the blood vessels in four nasal polyps stained orange red. Hyalinized collagen in six specimens. and the aural fibroma showed moderate affinity for $\overrightarrow{\vec{\omega}}$ the stain; mucin, keratin, and hyalinized basement $\stackrel{\circ}{\circ}$ membranes were negative.

CONGO RED AFFINITY BY FREUDENTHAL'S METHOD All specimens containing the various types of hyaline $i$ stained in varying shades of orange-red. Normal $\vec{\omega}$ tissue components such as collagen, smooth muscle, 음 mucin, blood vessels, and keratin were also strongly stained. Poor differentiation was obtained between $\bar{z}$ the amyloid and the surrounding tissues in the control sections.

POLARIZATION MICROSCOPY OF UNSTAINED SECTIONS Areas of hyalinized collagen and the aural fibroma showed strong birefringence in the unstained state. The hyalinized areas in the polyps showed weaker birefringence, which was partly due to some collagen fibres and to small areas of hyaline material which appeared as groups of fine short fibrils. The appearance of these small areas altered when the specimen was rotated between crossed polaroid filters; fibrils which were birefringent in one plane of polarized light became negative if rotated through an angle of $90^{\circ}$. By careful comparison of these areas in the van Gieson and P.T.A.H.-stained sections it was found that the birefringent fibrils in the small areas corresponded to the areas which were stained blue with P.T.A.H.

POLARIZATION MICROSCOPY OF CONGO RED-STAINED SECTIONS The hyaline deposits which were birefringent in an unstained state became negative after $\widetilde{N}$ staining with both Congo red methods. In all cases N the birefringence of the areas of fine fibrils (P.T.A.H. N positive) was abolished by the Highman's method even though there was no visible staining. Hyaline deposits which showed weak staining with Highman's method or overstained with Freudenthal's method $ळ$ gave no birefringence. In contrast areas of hyalinized collagen or collagen fibres became strongly birefringent with both methods, but hyalinized basement $\vec{D}$ membranes, mucin, and keratin remained negative. $\frac{\rho}{\mathbb{Q}}$

All birefringent specimens stained with Congo red $\varrho$ were rotated between crossed polaroid filters to obtain dichroic effects. Areas of hyalinized collagen 8 


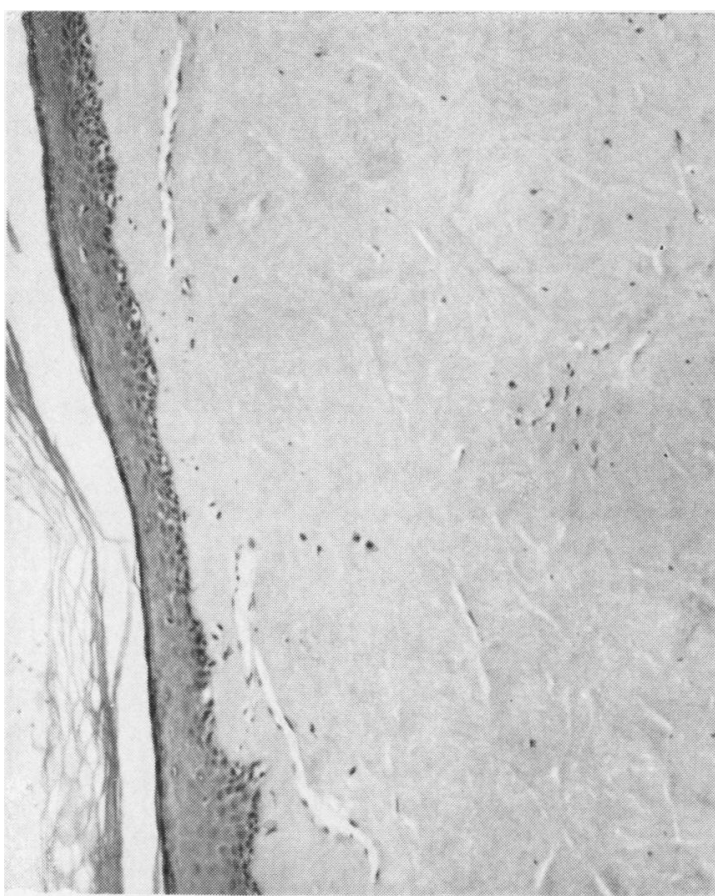

FIG. 1. Subepithelial hyalinized collagen which resembles amyloid. Haematoxylin and eosin $\times 100$.

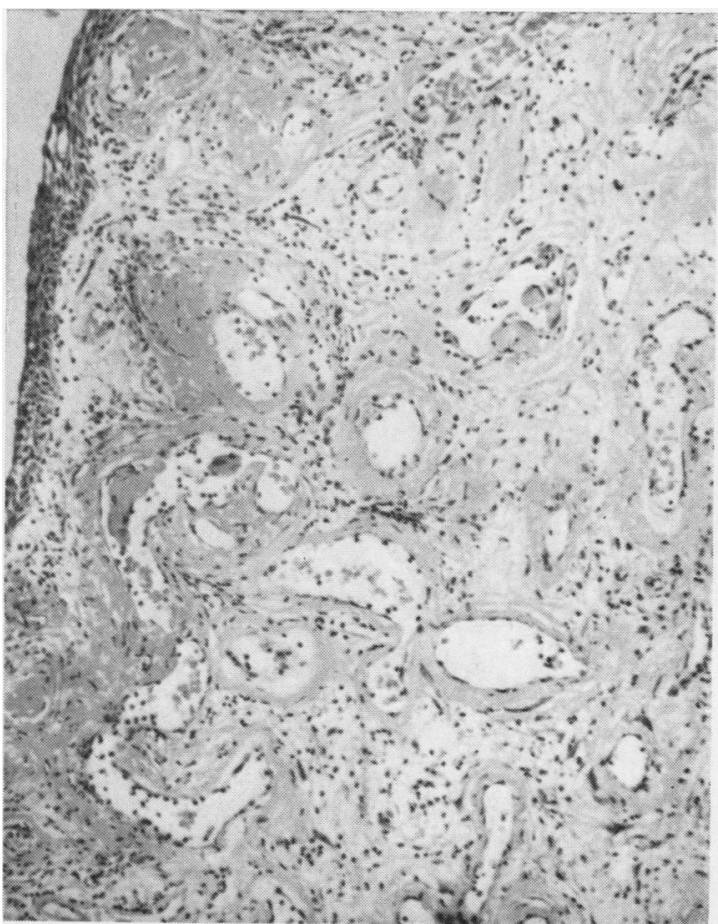

FIG. 3. Areas of perivascular and subepithelial hyaline deposits in a laryngeal polyp. Haematoxylin and eosin $\times 120$.

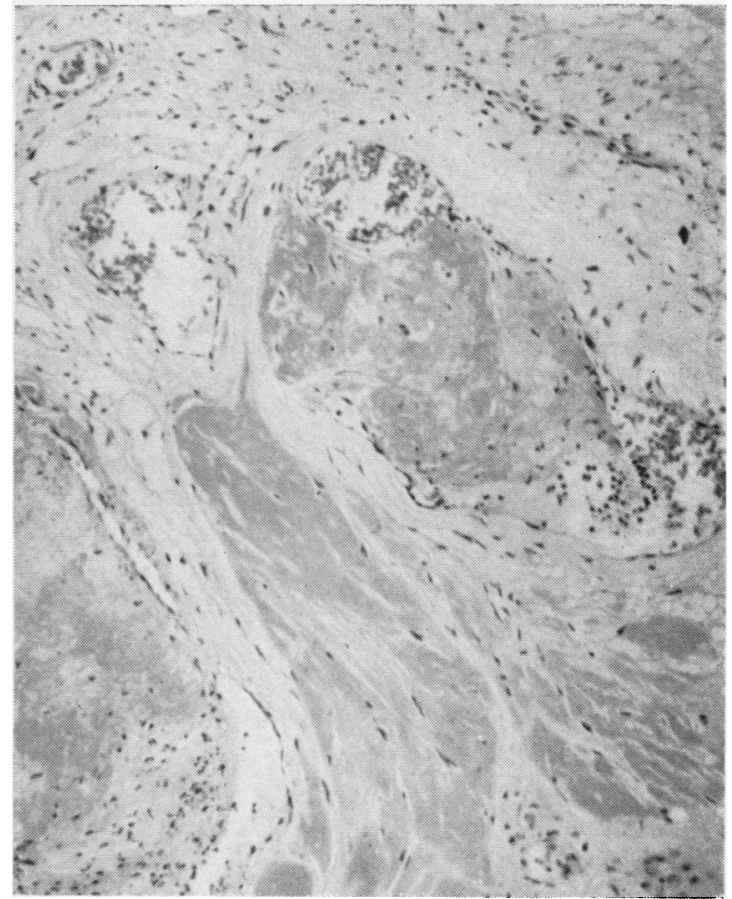

FIG. 2. Areas of interstitial and intravascular hyaline deposits in a laryngeal polyp. Haematoxylin and eosin $\times 100$.

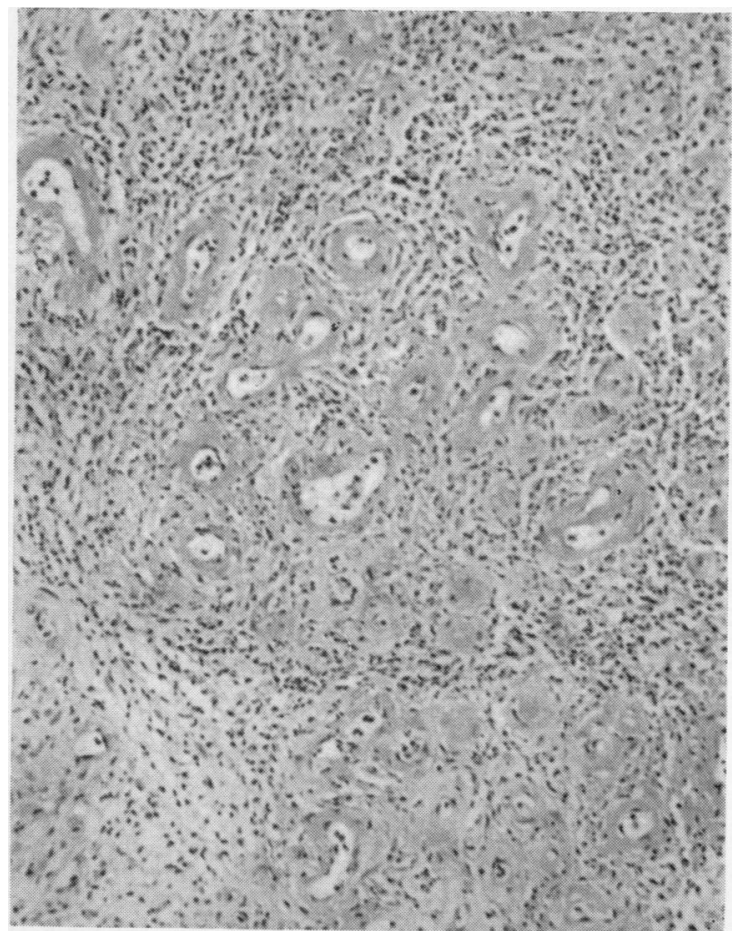

FIG. 4. Hyalinized vessels in an aural polyp. Haematoxylin and eosin $\times 120$. 
which were yellow-pink in one plane changed to green when rotated through a further angle of $90^{\circ}$. Some collagen fibres gave a pinkish-white and greenish-yellow colour. All the amyloid controls showed pink in one plane and green when rotated through a further $90^{\circ}$. The green colour obtained with amyloid was identical to that given by the Congo red-stained hyalinized collagen.

THIOFLAVIN T FLUORESCENCE One-third of the unstained hyaline deposits showed no auto-fluorescence, but the other two-thirds gave a dull purple-blue or grey-blue autofluorescence. The control amyloid deposits showed very little autofluorescence. Hyaline deposits stained with Thioflavin $\mathbf{T}$ only showed a slight difference when compared with the unstained sections. There was a slight increase of brilliancy, and the deposits gave a pale purple-blue or blue fluorescence. The aural fibroma and three specimens of hyalinized collagen showed much brighter fluorescence. Cartilage matrix gave a brilliant yellow fluorescence. The control amyloid deposits gave an overall brilliant silver-blue fluorescence when stained with Thioflavin $\mathrm{T}$, but the colour of the fluorescence was not homogenous as some specimens showed areas of bright blue or greenish-blue, with yellow or yellowishgreen hues. The superimposed colour varied for different specimens, but it was always constant for a particular specimen of amyloid. All the amyloid controls gave brilliant fluorescence except one specimen which had been kept in acid formalin for four years. It gave a dull blue fluorescence which was similar to that given by the hyaline deposits.

PEPSIN EXTRACTION TECHNIQUE Sections incubated in pepsin were compared with controls which had been incubated in hydrochloric acid without pepsin. The control sections showed slight or no loss of material in the hyaline deposits. However in 50 specimens the hyaline deposits were completely digested by the pepsin. In contrast the control

amyloid deposits, hyalinized basement membranes, and the fibroma showed slight or no digestion. Areas of hyalinized collagen showed about $50 \%$ loss of material.

PHOSPHOTUNGSTIC ACID HAEMATOXYLIN STAINING The areas of hyaline material in the laryngeal polyps all showed some areas staining dark blue, while other areas stained a reddish or purplish-brown. Considerable variation was obtained, as some $\vec{\circ}$ polyps showed areas of pale reddish-brown material containing a dark blue network of fibrils, while in other areas there were large masses or thick bands of dark blue material. In all the nasal and aural polyps ? the areas of interstitial hyaline or deposits related to blood vessels stained deep blue, but some deposits in $\omega$ vessel walls gave a purplish-red colour.

VAN GIESON'S STAIN The hyaline deposits in all the $\frac{9}{5}$ polyps stained a bright yellow (picric acid), but three specimens stained a khaki shade. Most specimens showed pink- or red- staining collagen fibres in or around the hyaline deposits. Areas of $\vec{\bullet}$ hyalinized collagen stained deep red or orange-red; hyalinized basement membranes stained orange-red.

\section{DISCUSSION}

The terms hyaline and hyalinization only describe the physical appearances of several different kinds of eosinophilic substances deposited or formed in tissues. These substances are not identical, but vary widely in their chemical composition which is still largely unknown. Amyloid is similar in appearance to these substances, but it can be distinguished from them by certain staining reactions. Hyaline is used to mean the non-amyloid group of eosinophilic substances in this study. The main differences between the staining reactions of the hyaline deposits and the amyloid controls are summarized and compared in Table II. Hyalinized collagen is included separately because it can be easily confused $I$ with amyloid infiltration in haematoxylin-eosin-

TABLE II

COMPARISON OF THE MAIN RESULTS

\begin{tabular}{|c|c|c|c|}
\hline \multirow[b]{2}{*}{ Method } & \multicolumn{2}{|c|}{ COMPARISUN UF THE MAIN RESULIS } & \multirow[b]{2}{*}{ Amyloid } \\
\hline & Hyaline Deposits & Hyalinized Collagen & \\
\hline Metachromasia & Negative & $\begin{array}{l}\text { Occasional areas of weak } \\
\text { metachromasia }\end{array}$ & Strong metachromasia \\
\hline Congo red (Highman's) & Low affinity & Moderate or high affinity & High affinity \\
\hline Polarized light, unstained & Weak birefringence & Strong birefringence & No birefringence \\
\hline Polarized light, Congo red stained & No birefringence & Strong birefringence & Strong birefringence \\
\hline Dichroism & Negative & Positive & Positive \\
\hline Thioflavin $\mathrm{T}$, fluorescence & Weak & Weak or moderate & Strong \\
\hline Pepsin extraction & Complete digestion & Partial digestion & No significant digestion \\
\hline P.T.A.H. & $\begin{array}{l}\text { Dark blue areas, or network } \\
\text { with purplish-brown or reddish- }\end{array}$ & Reddish-brown & Pale reddish-brown \\
\hline van Gieson & $\begin{array}{l}\text { brown areas } \\
\text { Bright yellow }\end{array}$ & Red or orange-red & Greenish-yellow \\
\hline
\end{tabular}


stained sections and by only using some of the special methods given in Table II. The staining reactions of the hyaline substances did not satisfy the criteria described as being characterstic of amyloid by McAlpine et al. (1963). The exact identity of the hyaline substances (except for fibrin) remains unknown, but their staining reactions fell into a group which is quite different from amyloid or hyalinized collagen (Table II). The results also indicate that any hyaline deposit which is suspected of being true amyloid must be studied by using all the methods or stains.

The metachromatic staining reaction of amyloid is used histologically to distinguish it from other hyaline materials. It is significant that no hyaline deposits in the polyps showed this staining reaction; also the few polyps which showed some staining with Highman's Congo red method were not metachromatic. Epstein, Winston, Friedmann, and Ormerod (1957) found no metachromatic staining in hyalinized vocal cord polyps which showed some affinity for Congo red. Kelly and Craik (1952) omitted methyl violet metachromasia from their study of hyalinized laryngeal polyps on the grounds that the stain was not permanent. It must be stressed here that metachromasia must always be assessed on any homogeneous eosinophilic deposit or hyalinized material suspected of being amyloid, even though the method does suffer from impermanence.

Although Congo red was introduced by Bennhold (1922) as a stain for amyloid, Ladewig (1945), Stark and McDonald (1948), Dahlin (1949), Montgomery and Muirhead (1954), Symmers (1956), Montgomery and Muirhead (1957), Pearse (1960), and McAlpine et al. (1963) all reported non-specific staining reactions of various normal and pathological tissue constituents, particularly hyaline deposits and hyalinized collagen. In the present study most of the hyaline deposits in the polyps were negative when using Highman's technique (1946), or a few showed a low affinity for Congo red, but specimens of hyalinized collagen showed a moderate affinity for the stain. Further, four nasal polyps showed Congo-red-stained vessels with this technique, but these were not metachromatic and stained blue or purplish-red with phosphotungstic acid haematoxylin. Hyaline deposits in the polyps show a high affinity for Congo red with other staining methods employing aqueous solutions of the dye, such as Freudenthal's technique, and hence can be confused with amyloid. The alcoholic solution of Congo red, as used in Highman's method, gives some degree of selectivity between hyaline and amyloid, but even the results of this better method should be interpreted with caution and used in conjunction with other methods.
Ladewig (1945) reported that Congo-red-stained amyloid became birefringent when examined with polarized light. He also reported that other nonamyloid substances (hyalines, mucins, fibrinoid, and elastic tissue), which also stain with the dye, showed no birefringence. In the present study it is significant that all hyaline deposits stained by Freudenthal's method, or those which showed weak staining with Highman's method, gave no birefringence or dichroism in polarized light. Many of these deposits showed some intrinsic birefringence which was probably due to collagen, reticulin fibres, or fibrin. It is interesting that this intrinsic birefringence was abolished or diminished by both Congo red methods, and, in the case of Highman's method, without visible staining. In contrast the intrinsic birefringence of hyalinized collagen was increased by staining with both Congo red methods, but to a lesser degree with Highman's technique. This emphasizes the difficulty of detecting amyloid deposits in masses of hyalinized collagen, particularly when the Congored-stained collagen shows similar dichroism as amyloid.

Unstained sections of laryngeal, aural, and nasal polyps showed slight autofluorescence when examined in ultra-violet light. After staining with Thioflavin $\mathrm{T}$ there was a slight increase of brilliancy which was more marked in the case of three specimens of hyalinized collagen. These findings differ from those for amyloid which showed brilliant fluorescence when stained with this fluorochrome. The overall colour obtained with amyloid was a brilliant silver-blue, but this colour was not homogeneous. Areas of either bright blue or greenish-blue with yellow or yellowish-green fluorescence were obtained with different specimens. Vassar and Culling (1959), who first applied this fluorochrome to amyloid, failed to give any indication of the colour of the fluorescence, except to say that specimens from primary amyloidosis gave a brighter fluorescence. Kurban (1960) reported that amyloid deposits in the skin gave a bright light blue fluorescence, and that hyaline deposits in cases of colloid milum and senile elastosis gave no fluorescence. Vassar and Culling (1961), in a report claiming that amyloid deposits occur in cases of thyroid carcinoma, state that the material suggestive of amyloid gave a brilliant yellow-green fluorescence. In another report Vassar and Culling (1962) only regarded a yellow or yellow-green fluorescence as being significant of amyloid. Independently Peterson and Schulz (1961) described a case of amyloid deposits in the vessel walls of a vascular malformation of the brain which gave a brilliant yellow fluorescence with the Thioflavin $\mathrm{T}$ method. All reports are in agreement that amyloid fluoresced brilliantly when 
stained with this fluorochrome. The disagreement on the colour of the fluorescence may reflect differences in batches of dye, ultra-violet light wavelengths, fixation and processing of the tissues, or the composition of amyloid. Vassar and Culling (1961 and 1962) did not compare the Thioflavin $T$ fluorescence with other stains for amyloid, but Kurban (1960) and Peterson and Schulz (1961) supported their observations with other amyloid staining methods. In the present study the different colours of fluorescence were given by amyloid specimens which had been studied by all the other methods given in Table II, so there can be no doubt about the identity of the amyloid. Hence it appears that ultra-violet light can excite strong blue, green, or yellow wavelengths, as pure or mixed colours from amyloid stained with Thioflavin $\mathrm{T}$, and further that green or yellow wavelengths are not obtained from specimens of hyaline deposits. Although the fluorescence was brighter in the specimens of hyalinized collagen, the colour obtained was light blue. Difficulties of identifying deposits are likely to occur with this method if strong blue fluorescence is obtained from hyalinized collagen, excitation of green or yellow wavelengths from non-amyloid materials, e.g., cartilage matrix, and quenching or alteration of the fluorescence by prolonged fixation. In the present study the Thioflavin $\mathrm{T}$ method was found to be valuable in distinguishing amyloid from hyaline deposits, but it should only be used to identify amyloid in conjunction with other methods.

Hyaline deposits in laryngeal, aural, and nasal polyps were digested by pepsin, but the amyloid controls were not significantly affected. Missmahl (1950), Wagner (1957), and Arvy and Sors (1958) reported that amyloid was resistant to the digestion of proteolytic enzymes. Windrum and Kramer (1957) showed that amyloid was relatively more resistant to peptic digestion than either collagen or scar tissue. Pearse (1960) remarked that enzyme digestion is not usually used as a diagnostic procedure. McAlpine et al. (1963) found it useful to distinguish hyaline deposits in a tonsil and a laryngeal polyp which were suspected of being amyloid. The present study also shows that this method is of diagnostic value in distinguishing amyloid from hyaline (non-amyloid) deposits.

Although considerable variation was obtained in the staining pattern of laryngeal, aural, and nasal polyps with the P.T.A.H. stain they all showed some blue-staining areas of fibrin. In contrast no fibrin was found in the amyloid deposits in cases of localized amyloidosis (McAlpine et al., 1963; McAlpine and Fuller, 1964). This finding is interesting because it probably indicates a vascular origin for the hyaline deposits found in the polyps. No differentiation is possible between the hyalinized $\frac{\stackrel{0}{*}}{5}$ collagen which resembles amyloid, and amyloid with $\stackrel{-}{-}$ the P.T.A.H. stain, except that the amyloid generally $\underset{\vec{D}}{\vec{D}}$ stains a pale reddish-brown.

Symmers (1956) and Dahlin (1949) reported that a 응 van Gieson stain was particularly useful in distin- $\frac{\partial}{\bar{c}}$ guishing amyloid from hyalinized collagen. Amyloid $\vec{\nabla}$ deposits stain a pale greenish-yellow or khaki colour $\varrho$ with this stain. The hyaline material in the polyps कै showed a strong affinity for the picric acid com- $\overrightarrow{0}$ ponent of the stain but three specimens also stained $\overrightarrow{-}$ the same colour as amyloid. Coagulated serum $\vec{\sigma}$ proteins, present in the lumina of blood vessels, $\frac{\Omega}{0}$ also showed strong affinity for the picric acid. The specimens containing hyalinized collagen were $\vec{v}$ fuchsinophilic, staining red or orange-red. These $\omega$ results show that a van Gieson stain is essential to $\vec{\omega}$ detect hyalinized collagen which may closely resemble amyloid, and that all non-fuchsinophilic deposits must be studied by other methods in order to determine their nature.

No areas of amyloid were found in the hyaline deposits of the laryngeal polyps. Ash and Schwartz (1944), Ash and Raum (1956), Molnár, Mérei, and $\stackrel{+}{+}^{\circ}$ Szlepka (1957), and Fini Storchi (1957) all found no evidence of amyloid in hyalinized laryngeal polyps. However, Falbe-Hansen (1955) described the occurrence of laryngeal polyps in two patients a few years before the development of localized amyloid- $\stackrel{\mathbb{Q}}{\complement}$ osis, but a detailed report of the histology and $\overrightarrow{\vec{F}}$ staining characteristics was omitted. Although $\frac{0}{3}$ Cameron (1952) mentions that hyaline may accompany amyloid, there are no reports in the literature which describe areas of amyloid occurring in a hyalinized laryngeal polyp. The larynx is unique in? respect that it is the commonest site in the body to be $\frac{0}{3}$ affected by the uncommon condition of localized amyloidosis (Pollak, 1914; Rey, 1937; McAlpine $ᄋ$ and Fuller, 1964); also it is the site of the relatively more common condition of hyalinized polyps. 을 These conditions are regarded as two different $D$ pathological entities, but they affect the same? tissues, so if amyloid is formed from hyaline substances then the larynx is the best site in which to study this possible phenomenon. McAlpine and ${ }^{\circ}$ Fuller (1964) investigated a patient with localized laryngeal amyloidosis who had 'polypoidal mucosa' removed from the larynx 10 years before presenta-e tion with amyloid deposits, but re-examination of the earlier biopsy material only showed the presence? of amyloid which had been previously overlooked. T] Further work is required on other cases if this type, $\frac{\vec{C}}{0}$ using the methods described in the present report, $\stackrel{\mathbb{Q}}{\stackrel{Q}{\overparen{Q}}}$ so as to study the theoretical possibility of hyaline $\mathbb{\Omega}_{\square}$ deposits being converted into amyloid, and tog establish that the formation of amyloid or hyaline 
substances in the larynx are by different pathological processes.

We should like to thank Professor I. Friedmann for the histological specimens and laboratory facilities, Mr. M. Bohler for technical assistance, and Mr. R. Tunnicliffe for the photomicrographs.

\section{REFERENCES}

Arvy, L., and Sors, C. (1958). Acta histochem. (Jena), 6, 77.

Ash, J. E., and Schwartz, L. (1944). Trans. Amer. Acad. Ophthal. Otolaryng., 48, 323.

-_, and Raum, M. (1956). An Atlas of Otolaryngic Pathology. Armed Forces Institue of Pathology, Washington.

Bennhold, H. (1922). Münch. med. Wschr., 2, 1537.

Cameron, G. R. (1952). Pathology of the Cell. Oliver \& Boyd, Edinburgh,

Culling, C. F. A. (1957). Handbook of Histopathological Tec/mique. Butterworth, London.

Dahlin, D. C. (1949). Amer. J. Path., 25, 105.

Epstein, S. S., Winston, P., Friedmann, I., and Ormerod, F. G. (1957). J. Laryng., 71, 673.
Falbe-Hansen, J. (1955). Acta oto-laryng. (Stockh.), 45, 388.

Fini Storchi, O. (1957). Boll. Mal. Orecch., 75, 252.

Highman, B. (1946). Arch. Path., 41, 559.

Kelly, H. D. Brown, and Craik, J. E. (1952). J. Laryng., 66, 339.

Kurban, A. K. (1960). Bull. Johns Hopk. Hosp., 107, 320.

Ladewig, P. (1945). Nature (Lond.), 156, 81.

McAlpine, J. C., and Fuller, A. P. (1964). J. Laryng., 78, 296.

$\longrightarrow$, Radcliffe, A., and Friedmann, I. (1963). Ibid., 77, 1.

Missmahl, H. P. (1950). Virchows Arch. path. Anat., 318, 518.

Molnár, L., Mérei, G., and Szlepka, G. A. (1957). Fül-Orr-Gégegyóg. $1,17$.

Montgomery, P. O’B., and Muirhead, E. E. (1954). Amer. J. Path., 30,521

(1957). Ibid., 33, 285.

Pearse, A. G. E. (1960). Histochemistry, Theoretical and Applied, 2nd ed. Churchill, London.

Peterson, E. W., and Schulz, D. M. (1961). Arch. Path., 72, 480.

Pollak, E. (1914). Z. Laryng. Rhinol., 7, 25.

Rey, W. (1937). Arch. Ohr.-, Nas.-, u. Kehlk.- Heilk., 143, 216.

Stark, D. B., and McDonald, J. R. (1948). Amer. J. clin. Path., 18, 778.

Symmers, W. St. C. (1956). J. clin. Path., 9, 187.

Vassar, P. S., and Culling, C. F. A. (1959). Arch. Path., 68, 487.

- - - (1961). Amer. J. clin. Path., 36, 244.

- - (1962). Arch. Path., 73, 59.

Wagner, B. M. (1957). In Analvtical Pathology, edited by R. C. Mellors, p. 429. McGraw-Hill, New York.

Windrum, G. M., and Kramer, H. (1957). Arch. Path., 63, 373. 